Bryn Mawr College

Scholarship, Research, and Creative Work at Bryn Mawr

College

Physics Faculty Research and Scholarship

Physics

1996

\title{
Excitation of an Atomic Electron to a Coherent Superposition of Macroscopically Distinct States
}

Michael W. Noel

Bryn Mawr College, mnoel@brynmawr.edu

C. R. Stroud Jr.

Let us know how access to this document benefits you.

Follow this and additional works at: http://repository.brynmawr.edu/physics_pubs

Part of the Physics Commons

\section{Custom Citation}

Michael W. Noel and C. R. Stroud, Jr., "Excitation of an Atomic Electron to a Coherent Superposition of Macroscopically Distinct States," Phys. Rev. Lett. 77, 1913 (1996).

This paper is posted at Scholarship, Research, and Creative Work at Bryn Mawr College. http://repository.brynmawr.edu/physics_pubs/77

For more information, please contact repository@brynmawr.edu. 


\title{
PHYSICAL REVIEW \\ LETTERS
}

\section{Excitation of an Atomic Electron to a Coherent Superposition of Macroscopically Distinct States}

\author{
Michael W. Noel and C. R. Stroud, Jr. \\ The Institute of Optics, University of Rochester, Rochester, New York 14627
}

(Received 15 November 1995)

\begin{abstract}
An atomic electron is prepared in a state closely analogous to Schrödinger's coherent superposition of "live cat" and "dead cat." The electronic state is a coherent superposition of two spatially localized wave packets separated by approximately $0.4 \mu \mathrm{m}$ at the opposite extremes of a Kepler orbit. State-selective ionization is used to verify that only every other atomic level is populated in the "cat state," and a Ramsey fringe measurement is used to verify the coherence of the superposition. [S0031-9007(96)01071-X]
\end{abstract}

PACS numbers: 03.65.Bz, 32.80.Rm, 42.50.Md

Because the description of reality appears so different in classical and quantum physics, the boundary between the macroscopic and microscopic worlds has long been a fascinating area of research. Schrödinger brought the distinction between the two descriptions into sharp focus by introducing a cat that was placed in a quantum superposition of "live cat" and "dead cat" [1].

Schrödinger's cat has been the subject of an enormous number of papers and books [2], and has even become familiar to the layman. With recent developments in technology in the mesoscopic regime (where dimensions are of the order of a micron) that lies at the boundary between the microscopic and macroscopic regimes, there have been proposals of practical systems that are realizations of Schrödinger's cat state. The systems considered vary greatly, from nonlinear optics $[3,4]$ and micromasers $[5,6]$ to molecules $[7,8]$ and trapped ions [9].

In these realizations a system with macroscopic (or nearly macroscopic) dimensions that is reasonably well modeled as a harmonic oscillator is excited into a superposition of two coherent states. This simple act of superposing two quasiclassical states results in some remarkably nonclassical features such as sub-Poissonian and oscillatory number state distributions, squeezing, and interference [10-12].

A superposition of two harmonic oscillator coherent states forms a Schrödinger cat state when the two are chosen to be localized at macroscopically separated points. A state of this type can be written

$$
|\psi(t)\rangle=N\left[|\alpha(t)\rangle+e^{i \phi}|-\alpha(t)\rangle\right],
$$

where $N$ is a normalization constant. The coherent states can be expanded out in terms of number states

$$
|\alpha(t)\rangle=e^{-|\alpha|^{2} / 2} \sum_{n=0}^{\infty} \frac{|\alpha(0)|^{n}}{\sqrt{n !}} e^{-i(n+1 / 2) \omega t}|n\rangle .
$$

In this case each of the coherent states forms a minimum uncertainty wave packet oscillating back and forth periodically between its turning points in a parabolic potential. There are two phases associated with this oscillation. The phase of the complex number $\alpha(0)$ determines the locations of the two wave packets at $t=0$. For real $\alpha(0)$, the two wave packets start out at the opposite extremes of the oscillatory motion separated by the distance $2 \alpha(0)$. During a single period of the oscillation the two wave packets will pass through each other as each goes through the same motion. The constant $\phi$ is the phase difference between the de Broglie waves associated with the two packets. When the two wave packets overlap this phase will determine the nature of the interference between them. As we will see later, it also determines the population distribution in the various eigenstates. At some particular time in the motion the system will in general be in a superposition which is simultaneously localized in two different locations. If the two positions are 
macroscopically distinguishable then this state is similar to Schrödinger's cat state in that it represents a quantum mechanical superposition of two classically distinguishable physical states which are localized in one or the other of the two classical positions only by the act of a measurement which destroys the superposition.

We have used two phase-coherent laser pulses to excite a single atomic electron into a state well approximated by Eq. (1), in which the electron is simultaneously localized in two wave packets each oscillating in and out about the nucleus in a single Kepler orbit. The diameter of the orbit is approximately $0.4 \mu \mathrm{m}$, which is not quite macroscopic, but mesoscopic, of the order of size of a transistor on a computer chip. We characterize the wave packet cat state in two ways. First, the quantum eigenstate distribution is measured for various phase differences between the two wave packets. Next, we probe the interference between the two wave packets to demonstrate the coherent, rather than statistical, nature of the superposition.

A radial wave packet is formed when an atom interacts with a short laser pulse tuned to the Rydberg series where the atomic energy levels are closely spaced [13-16]. The result of this interaction is the excitation of some of the population from the ground state to a coherent superposition of Rydberg states. The number of states in the superposition depends on the bandwidth of the transform limited laser pulse and, in the weak field limit, is simply the Fourier transform of the pulse envelope. Since the superposition is of energy eigenstates all with the same angular momentum, the localization of the electron is in the radial coordinate only. The electron (initially localized to a radial shell near the core) oscillates in and out in a breathing motion at the classical Kepler period. For short times a radial wave packet is very similar to the coherent state of a harmonic oscillator which also consists of a superposition of energy eigenstates [Eq. (2)] and results in a localized probability oscillating back and forth in the harmonic potential. In the harmonic oscillator this classical oscillation will continue indefinitely provided there is no further interaction after the initial excitation. Since the atomic potential is only approximately harmonic, the radial wave packet oscillates classically for only a limited period of time. The complex dynamics of atomic electron wave packets have been described in detail by several authors [17-20].

A Schrödinger cat state of the atom is formed by interacting with a pair of phase-coherent time-delayed laser pulses. The separation of these pulses is one-half of the Kepler period. The first pulse excites a portion of the population to a radial wave packet state. One-half of the Kepler period later the wave packet excited by the first pulse has moved to the outer turning point and the second laser pulse arrives to excite another wave packet at the inner turning point. The phase between the two laser pulses is very carefully controlled. The result of this sequence is that a single electron is now in a superposition of being at two well separated spatial locations (the inner and outer turning points).

We set up the experiment as follows. A $25 \mathrm{ps}, 10 \mu \mathrm{J}$, nearly transform-limited laser pulse was tuned to couple the ground state of a potassium atom to the Rydberg series. This pulse was divided into two time-delayed pulses with a beam splitter and pair of delay lines. We stabilized and controlled the time delay between the two pulses with an active servo system. The pulse pair was then directed onto a potassium atomic beam where we excited the radial wave packet cat state.

The quantum state distribution was measured for various phase differences between the two wave packets. The presence of a second wave packet modifies the state distribution compared to that of a single wave packet. This is one of the signatures of this type of coherent superposition state. Two particularly dramatic state distributions occur for the so called even and odd coherent states, $|\alpha\rangle+|-\alpha\rangle$ and $|\alpha\rangle-|-\alpha\rangle$, respectively. The even coherent state contains only even eigenstates $|n\rangle$, and the odd state is a superposition of only the odd eigenstates.

We use state-selective field ionization to measure the quantum state distribution of our atomic cat state. This technique consists of ramping on a dc electric field which ionizes the population from different Rydberg states at different times as each state's ionization potential is reached during the ramp. The ions are collected with an electron multiplier and binned according to their arrival time. The resulting time-resolved ion signal has peaks which correspond directly to different Rydberg energy eigenstates in the superposition. The time bins in our experiment are $50 \mathrm{~ns}$ wide and the peak separation varies from about $200 \mathrm{~ns}$ to $1 \mu \mathrm{s}$. These peaks are not equally spaced in time since the ionization potential goes as $1 / 16 n^{4}$. Each peak also has a width due to field inhomogeneities and geometrical averaging in the interaction region. The population in a given Rydberg state is the integrated signal in the peak associated with that state.

Figure 1(a) shows a reference trace taken with one delay arm blocked. This is the state distribution for a single wave packet. There are about five states in the superposition centered near $\bar{n}=66$. Next, we measured the distribution for excitation with the pulse pair. In Fig. 1(b) the phase between pulses was set to eliminate population from the odd Rydberg states. This mimics the state distribution for the even harmonic oscillator cat state. The phase can also be set to eliminate population from the even Rydberg states [Fig. 1(c)]. This is similar to the odd harmonic oscillator cat state.

The modification of the population distribution is a direct indication of the interference between the two quasiclassical wave packets. Another characteristic feature of Schrödinger's cat state is the interference between the 


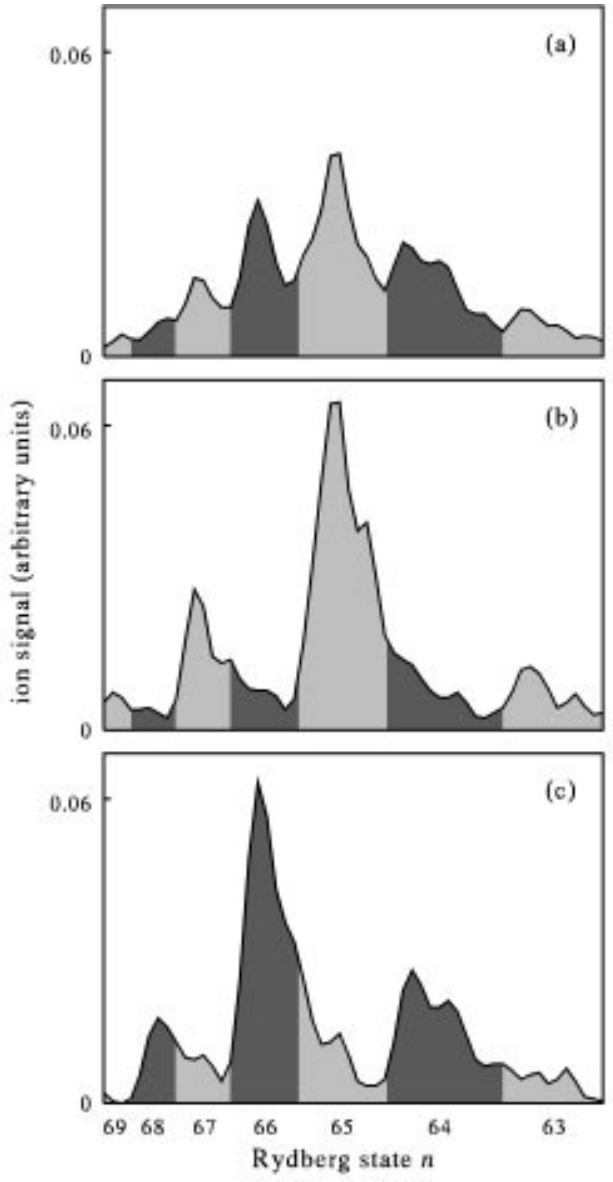

FIG. 1. Quantum state distribution of the radial wave packets measured using state-selective field ionization. Wave packets excited with (a) a single laser pulse, (b) a pulse pair of phase difference $\pi$, and (c) a pulse pair of phase difference 0 . Each of the peaks in these time-resolved ion signals correspond directly to a given Rydberg state in the superposition. The odd Rydberg states are shaded light gray, and the even states are shaded dark gray.

two wave packets in phase space. We have looked at this phase space interference at a time when the two wave packets spatially overlap to demonstrate the coherent rather than statistical nature of the superposition. In a statistical mixture the wave packets behave like ensembles of classical particles and simply add incoherently. However, if the superposition is coherent, the wave packets can interfere, and their wave nature is revealed. The interference between a pair of coherently excited wave packets was recently described as a type of Young's double slit interferometer [21]. Here we measure the interference in a similar way by probing with a third short laser pulse.

The location of a radial electron wave packet can be determined by interacting with a time-delayed probe laser pulse. If the wave packet is near the core when the probe pulse arrives, there is a strong interaction between the two. On the other hand, if the wave packet is away from the core when the probe pulse arrives, the interaction between the two is very weak. If the probe pulse is identical to the pulse which originally excited the wave packet, the two can interact coherently [22]. In this case a type of Ramsey fringe is observed (rapid oscillation in the excited state population at the optical period). The amplitude of the Ramsey fringes is large when the wave packet is near the core where it can interact with the probe pulse, and small when the wave packet is away from the core.

To measure the interference between the wave packets in our atomic Schrödinger cat state we must look at a time when the two wave packets spatially overlap and are near the core, where the probe interacts strongly. Initially the two wave packets are well separated and do not overlap at all near the core. However, since the atomic potential is not harmonic, the wave packets do not remain well localized, but spread as they oscillate. We probe at a fixed time in the evolution when the wave packets have spread enough so that their overlap near the core is very good. The Ramsey fringe amplitude is plotted as a function of the phase difference between the two wave packets in the cat state in Fig. 2. The excellent fringe visibility indicates two things. First, the wave packets must be in a coherent superposition to interfere at all, and second, the probe was in fact fixed at a time when the wave packets were well overlapped so they could strongly interfere.

The Schrödinger cat state which we have excited in the atom displays many of the nonclassical features which result from the superposition of two quasiclassical states. Although the extent of our wave packet's orbit is enormous compared to the size of a ground state atom and its behavior very nearly that of a classical planetary system (at least for short times) it is still far from being a living, breathing macroscopic cat.

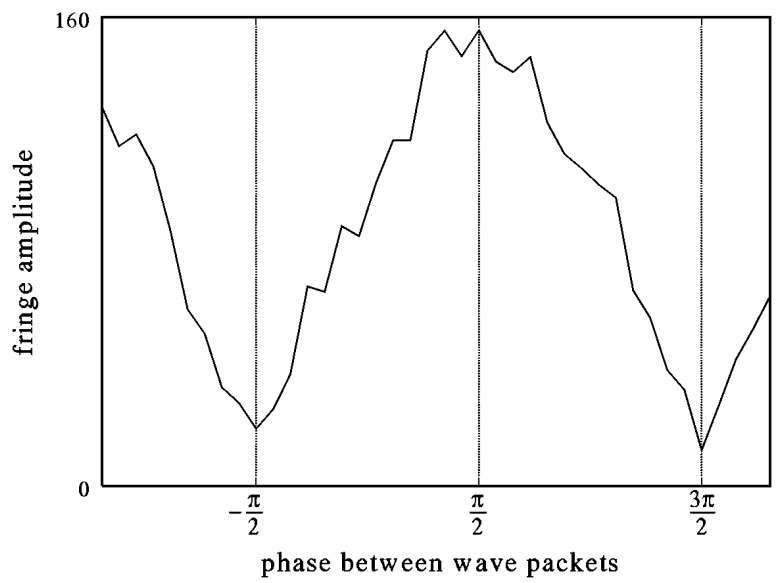

FIG. 2. Schrödinger cat state probed with a third time-delayed laser pulse. The time delay of the probe pulse is fixed at ten Kepler periods after the initial excitation. At this time the two wave packets have spread enough that their spatial overlap is very good. The Ramsey fringe amplitude is measured as a function of the phase difference between the two wave packets in the cat state revealing strong interference between the two. 
A cat has other macroscopic properties in addition to size. In particular, it has many degrees of freedom whereas the linear harmonic oscillator, no matter how large, has only one degree of freedom. One might argue that the number of degrees of freedom is the essential feature distinguishing the microscopic from the macroscopic. However, we note that a protein molecule has many degrees of freedom and yet it remains fundamentally a quantum object. Many definitions have been offered of the classical limit, and it is not clear that any of them suffices for all cases. Until we can actually achieve a quantum superposition involving indisputably macroscopic objects like cats, it is perhaps interesting to investigate, as we have, quantum superpositions of states of objects which are at least of dimensions approaching the macroscopic.

In a perfectly isolated quantum system the size of our cat state could be increased to truly macroscopic dimensions, all the while maintaining its quantum coherence. However, in any real experimental situation this complete isolation is of course impossible. The ways in which these environmental interactions lead to the decoherence of a macroscopic superposition vary depending on the quantum system of interest are some of the motivations for studying such states $[23,24]$. One way in which the environment couples to our atomic electron cat state is through the interaction with blackbody radiation. The time scale for mixing due to blackbody transitions goes as $n^{2}$ while the Kepler period scales as $n^{3}$ [25]. So, as the wave packets are excited higher in the Rydberg series creating more macroscopic superpositions there is a point at which the coherence between them will be lost before the wave packets can complete even a single orbit.

An atomic electron wave packet which was localized at two well-separated spatial positions was observed several years ago by Yeazell and Stroud [19]. This is the form that results when a single atomic wave packet is allowed to evolve freely to what is referred to as the one-half fractional revival [26]. In this paper we have shown that the one-half fractional revival is a Schrödinger cat state, and by exciting it directly with a pair of laser pulses we were able to control the phase between the two wave packets and more fully characterize the peculiar, nonclassical properties of the Schrödinger cat state.

We would like to acknowledge helpful discussions with I. A. Walmsley, J. Bromage, M. F. Van Leeuwen, John D. Corless, and James A. West. This work was supported in part by the Army Research Office.

Note added.-A Schrödinger cat state has recently been excited with a single ion in a trap [27].

[1] E. Schrödinger, Naturwissenschaften 23, 807 (1935); 23,
823 (1935); 23, 844 (1935); J.D. Trimmer, Proc. Am. Philos. Soc. 124, 323 (1980).

[2] John R. Gribbin, In Search of Schrödinger's Cat: Quantum Physics and Reality (Bantam Books, Toronto, 1984).

[3] B. Yurke and D. Stoler, Phys. Rev. Lett. 57, 13 (1986).

[4] Shang Song, Carlton M. Caves, and Bernard Yurke, Phys. Rev. A 41, 5261 (1990).

[5] Pierre Meystre, John J. Slosser, and Martin Wilkens, Opt. Commun. 79, 300 (1990).

[6] M. Brune, S. Haroche, J. M. Raimond, L. Davidovich, and N. Zagury, Phys. Rev. A 45, 5193 (1992).

[7] J. Janszky, An. V. Vinogradov, and T. Kobayashi, Phys. Rev. A 50, 1777 (1994).

[8] I. A. Walmsley and M.G. Raymer, Phys. Rev. A 52, 681 (1995).

[9] R.L. de Matos Filho and W. Vogel, Phys. Rev. Lett. 76, 608 (1996).

[10] U.M. Titulaer and R.J. Glauber, Phys. Rev. 145, 1041 (1966).

[11] W. Schleich, M. Pernigo, and Fam Le Kien, Phys. Rev. A 44, 2172 (1991).

[12] V. Bužek, A. Vidiella-Barranco, and P.L. Knight, Phys. Rev. A 45, 6570 (1992).

[13] Jonathan Parker and C. R. Stroud, Jr., Phys. Rev. Lett. 56, 716 (1986).

[14] G. Alber, H. Ritsch, and P. Zoller, Phys. Rev. A 34, 1058 (1986).

[15] A. ten Wolde, L.D. Noordam, A. Lagendijk, and H.B. van Linden van den Heuvell, Phys. Rev. Lett. 61, 2099 (1988).

[16] John A. Yeazell, Mark Mallalieu, Jonathan Parker, and C. R. Stroud, Jr., Phys. Rev. A 40, 5040 (1989).

[17] I. Sh. Averbukh and N.F. Perelman, Phys. Lett. 139A, 449 (1989).

[18] Z.D. Gaeta and C.R. Stroud, Jr., Phys. Rev. A 42, 6308 (1990).

[19] John A. Yeazell and C.R. Stroud, Jr., Phys. Rev. A 43, 5153 (1991).

[20] D. R. Meacher, P.E. Meyler, I. G. Hughes, and P. Ewart, J. Phys. B 24, L63 (1991).

[21] Michael W. Noel and C.R. Stroud, Jr., Phys. Rev. Lett. 75, 1252 (1995).

[22] L.D. Noordam, D.I. Duncan, and T.F. Gallagher, Phys. Rev. A 45, 4734 (1992).

[23] Juan Pablo Paz, Salman Habib, and Wojciech H. Zurek, Phys. Rev. D 47, 488 (1993).

[24] Wojciech H. Zurek, Salman Habib, and Juan Pablo Paz, Phys. Rev. Lett. 70, 1187 (1993).

[25] Thomas F. Gallagher, Rydberg Atoms (Cambridge University Press, New York, 1994).

[26] This is similar to the way a cat state is formed in a nonlinear optical system [3]; by injection of a coherent state into a nonlinear medium which causes the wave packet to disperse and reform as two or more separated wave packets.

[27] C. Monroe, D.M. Meekhof, B.E. King, and D.J. Wineland, Science 272, 1131 (1996). 